\title{
La politique extérieure de la démocratie portugaise
}

Mr Nuno Severiano Teixeira

\section{Citer ce document / Cite this document :}

Teixeira Nuno Severiano. La politique extérieure de la démocratie portugaise. In: Pôle Sud, n²2, 2005. pp. 63-74;

doi : 10.3406/pole.2005.1220

http://www.persee.fr/doc/pole_1262-1676_2005_num_22_1_1220

Document généré le 10/06/2016 


\title{
Résumé
}

Le processus de démocratisation au Portugal a affecté la politique intérieure mais également la politique extérieure. Le Portugal démocratique définit non seulement un nouveau modèle de société et d'institutions politiques mais aussi un nouveau modèle d'insertion internationale du pays. L'objectif fondamental de cet article est de proposer une approche de la dimension internationale de la transition et la consolidation de la démocratie au Portugal. Une première réflexion, dans une perspective de longue durée, aborde les modèles historiques d'insertion internationale du Portugal. Une deuxième réflexion aborde, la dimension internationale de la démocratisation portugaise entre 1974-1986. Finalement, la conclusion caractérise les lignes d'orientations stratégiques de la politique extérieure portugaise et, dans une perspective de continuité-changement, soutient que la démocratie introduit un nouveau modèle d'insertion internationale.

\begin{abstract}
The process of democratization in Portugal has affected its foreign policy as well as its domestic politics. Democratic Portugal has defined for itself not only a new model of society and political institutions, but also a new model for its participation in international affairs. The fundamental objective of this article is to formulate an approach to understanding the international dimension of the democratic transition and consolidation in Portugal. A first area of reflection, in the context of the long term, considers historical models of the international role of Portugal. This is followed by consideration of the international dimension of democratization between 1974 and 1986. A final section presents the main strategic orientations of contemporary Portuguese foreign policy in the perspective of continuity and change, and concludes that democracy has indeed introduced a new model of participation in international affairs for Portugal
\end{abstract}




\title{
La politique extérieure de la démocratie portugaise
}

\author{
Nuno Severiano Teixeira \\ Université Nova de Lisbonne
}

\section{Résumé/Abstract}

Le processus de démocratisation au Portugal a affecté la politique intérieure mais également la politique extérieure. Le Portugal démocratique définit non seulement un nouveau modèle de société et d'institutions politiques mais aussi un nouveau modèle d'insertion internationale du pays. L'objectif fondamental de cet article est de proposer une approche de la dimension internationale de la transition et la consolidation de la démocratie au Portugal. Une première réflexion, dans une perspective de longue durée, aborde les modèles historiques d'insertion internationale du Portugal. Une deuxième réflexion aborde, la dimension internationale de la démocratisation portugaise entre 1974-1986. Finalement, la conclusion caractérise les lignes d'orientations stratégiques de la politique extérieure portugaise et, dans une perspective de continuité-changement, soutient que la démocratie introduit un nouveau modèle d'insertion internationale.

The process of democratization in Portugal has affected its foreign policy as well as its domestic politics. Democratic Portugal has defined for itself not only a new model of society and political institutions, but also a new model for its participation in international affairs. The fundamental objective of this article is to formulate an approach to understanding the international dimension of the democratic transition and consolidation in Portugal. A first area of reflection, in the context of the long term, considers historical models of the international role of Portugal. This is followed by consideration of the international dimension of democratization between 1974 and 1986. A final section presents the main strategic orientations of contemporary Portuguese foreign policy in the perspective of continuity and change, and concludes that democracy has indeed introduced a new model of participation in international affairs for Portugal.

\section{2 \\ Mots-Clés/Keywords}

Atlantique, Démocratie, Europe, Politique extérieure, Relations post-coloniales Atlantic, Democracy, Europe, Foreign Policy, Post colonial Relations 
Le 25 avril 1974 et le processus de démocratisation dont il marque le début ont affecté la politique extérieure, mais également la politique intérieure du Portugal. Le Portugal démocratique définit non seulement un nouveau modèle de société et d'institutions politiques mais aussi un nouveau modèle d'insertion du pays dans le monde international, en accord avec le processus de démocratisation. En fait les quasi trente années de démocratie au Portugal se reflètent tout aussi clairement dans les structures internes que dans le positionnement international du pays. Cela signifie que la transition et la consolidation de la démocratie ont, sans aucun doute, une face internationale et une traduction dans la politique extérieure portugaise.

Dans le débat théorique sur la transition et la consolidation des démocraties, les premières études soutenaient la thèse selon laquelle le moteur fondamental de la démocratisation se centrait de façon prédominante sur les facteurs internes (Linz, Stepan, 1978; Schmitter, O'Donnell, Whitehead, 1986). Plus récemment, un ensemble d'études diverses ont attiré l'attention sur l'importance du facteur international dans les processus de démocratisation. Et, dans certaines de ces études, on s'efforce même de définir des modèles d'analyse globale dans le cadre de l'interdépendance structurelle croissante entre le contexte international et les facteurs internes dans le résultat final des processus de transition et de consolidation des démocraties (Huntington, 1991; Pridham, 1991, 2000; Pridham, Herring, 1997; Whitehead 1996; Schmitter, 1999).

Mon propos est ici plus modeste. Il ne s'agit pas d'une analyse à partir d'un modèle global, c'est-à-dire de l'impact du contexte international sur la transition et la consolidation démocratique, d'une part, et de l'impact des démocratisations sur le système des relations internationales, d'autre part, mais simplement d'une réflexion sur le facteur politique extérieure.

Dans le domaine limité de l'analyse de la politique extérieure, le débat scientifique sur les processus de démocratisation - transition et consolidation de la démocratie - tourne autour de deux questions fondamentales: premièrement, la dimension temporelle du processus de démocratisation dans la politique extérieure ou, en d'autres termes, la correspondance chronologique entre la transition et la consolidation au niveau intérieur et au niveau extérieur; deuxièmement, la dimension de rupture ou de continuité dans 
les politiques extérieures, avant et après l'avènement de la démocratie.

Pour tenter de répondre à ces questions je vais développer ma réflexion en deux étapes distinctes: en premier lieu, une réflexion sur les modèles d'insertion internationale du pays, à partir des conditions géopolitiques et des constantes historiques, c'est-à-dire des mouvements de longue durée qui déterminent les caractéristiques historiques de la politique extérieure portugaise; en second lieu, une réflexion sur la dimension internationale de la démocratisation, c'est-à-dire sur son évolution temporelle et sur le caractère de continuité ou de changement de la politique extérieure portugaise.

\section{Les modèles d'insertion internationale}

Pays européen, le Portugal est aussi et, simultanément, un pays atlantique. Petite puissance, semi-périphérique, le Portugal a encore une autre caractéristique géopolitique qui exercera un poids décisif sur la formulation de sa politique extérieure: il a une seule frontière terrestre et, de ce fait, il a toujours vécu en équilibre géopolitique instable entre le continent et la mer.

De ces constantes géopolitiques et de cette recherche continuelle d'un équilibre, découle un mouvement historique à long terme qui a déterminé, sinon des structures invariables, du moins, des lignes sous-tendant les options extérieures et les caractéristiques historiques de la politique extérieure portugaise et des modèles d'insertion internationale du Portugal.

Quels sont et comment se sont formés ces éléments de permanence historique dans la politique extérieure qui déterminent, à leur tour, les modèles d'insertion internationale du pays.
Le Portugal, historiquement, a connu trois modèles d'insertion internationale qui correspondent à trois moments différents.

Le premier modèle est celui du Portugal médiéval. Jusqu'au XVe siècle les relations extérieures du Portugal se déroulent dans le cadre de la péninsule Ibérique entre cinq unités politiques ayant toutes, plus ou moins, la même dimension et le même potentiel : les royaumes péninsulaires- Castille, Aragon, Léon, Navarre, et Portugal.

La lutte contre l'Islam, à l'intérieur de la péninsule, et les limitations au niveau de la science et de la technologie et des ressources rendaient par ailleurs impossibles toutes relations extra-péninsulaires durables. Au Moyen âge, les relations extérieures du Portugal se sont donc développées dans le cadre péninsulaire et dans un milieu international présentant un équilibre quasi naturel.

$\mathrm{Au} \mathrm{XV}$ siècle, ce cadre change radicalement et, c'est à partir de ce moment-là, qu'ont pris forme les conditions géopolitiques et les mouvements historiques à long terme de l'orientation extérieure du pays, lesquels ont déterminé le deuxième modèle d'insertion internationale du Portugal. Ce modèle s'est prolongé pendant cinq longs siècles et s'achève précisément avec le processus de démocratisation.

La victoire sur l'Islam et l'unification de l'Espagne pas les Rois Catholiques transforment la péninsule en deux unités de dimension et de potentiel inégal: le Portugal et l'Espagne.

D'autre part, l'évolution scientifique et technologique va permettre le développement durable de relations extra-péninsulaires.

De l'équilibre péninsulaire médiéval, on passe à un déséquilibre péninsulaire et, par voie de conséquence, à la recherche par le Portugal d'une compensation de ce déséquilibre. Compensation que sa façade atlantique et la 
possibilité de maintenir des relations extrapéninsulaires permettent d'ériger en " compensation maritime ». À partir de ce momentlà, le Portugal va vivre dans un effort constant pour trouver un équilibre entre la pression continentale de l'Espagne et la recherche d'une compensation maritime dans l'Atlantique (Macedo, 1987).

De cela découlent les éléments de permanence historiques des options stratégiques de la politique extérieure portugaise.

Premièrement, la perception d'une antinomie, qui va parfois jusqu'au dilemme entre le continent et la mer ou entre l'Europe et l'Atlantique.

Deuxièmement, et comme conséquence du premier élément, l'éloignement stratégique, par rapport à l'Europe et au Continent (perçu comme la menace de l'Espagne), et la prédominance du vecteur maritime dans l'orientation extérieure ou, en d'autres termes l'option Atlantique du Portugal.

Troisièmement, et comme conséquence du deuxième élément, deux mouvements historiques à long terme dans la politique extérieure portugaise: en premier lieu la recherche d'alliances privilégiées avec la puissance maritime (historiquement ce fut d'abord l'alliance anglaise, puis après la Première guerre mondiale, les États-Unis d'Amérique et, plus tard, sur un plan multilatéral, l'OTAN); en second lieu, le projet colonial s'est concrétisé dans plusieurs empires successifs - l'Inde, le Brésil, l'Afrique.

Quatrièmement, et comme conséquence des trois premiers points, la diversification des alliances extra-péninsulaires, en relation avec l'Espagne, et une diplomatie bilatérale, reposant sur le triangle Lisbonne-MadridLondres, et après 1945, Lisbonne-MadridWashington.

Ce sont ces lignes d'orientation qui ont donné forme à la politique extérieure de l'“ État Nouveau " de Salazar depuis 1935 jusquà la fin du régime (Teixeira, 1991).

Ces lignes sont clairement présentes déjà dans la première prise de position en matière de politique extérieure en 1935, avec la critique du parlementarisme international de la SDN, considérée comme centre politique continental et, en contrepoint, l'affirmation de la vocation atlantique du Portugal et la distanciation du Portugal des questions d'Europe centrale; la revalorisation des principes traditionnels de la politique extérieure portugaise avec la réaffirmation de l'Alliance Anglaise et de l'Amitié Péninsulaire; et enfin, la défense intransigeante de l'empire colonial.

Ce sont ces mêmes principes d'éloignement des questions européennes, d'affirmation d'un Portugal atlantique et colonial et du retour à l'équilibre du triangle LisbonneLondres-Madrid qui constitueront les orientations stratégiques de la politique extérieure portugaise pendant les années 30 et 40 , en particulier pendant la guerre civile espagnole (Delgado, 1981 ; Oliveira, 1987; Rosas, 1998) et pendant la deuxième guerre mondiale (Stone, 1994; Telo, 1987, 1991, 2000; Carrilho, 1989; Rosas, 1990), principes qui sappuient maintenant sur deux instruments diplomatiques bien définis: l'Alliance Anglaise et le Pacte Ibérique.

Et ce seront ces mêmes constantes historiques et ces mêmes lignes d'orientation stratégique qui vont persister dans l'après-guerre et présider aux positions internationales de Salazar, qui semble ne pas comprendre ou, en tout cas, ne pas accepter l'émergence d'un nouvel ordre international.

En premier lieu, le déclin de la GrandeBretagne et l'émergence des États-Unis comme nouvelle puissance maritime, qu'il ne reconnaît qu'avec l'entrée dans l'OTAN.

En deuxième lieu, la méfiance qu'il éprouvait à l'égard du parlementarisme de la SDN 
se manifeste, à nouveau, à l'égard de l'ONU en tant que nouvelle organisation à vocation mondiale.

En troisième lieu, il ne comprend pas que la reconstruction de l'Europe n'est plus possible dans le seul cadre national et doit, nécessairement, se faire dans le cadre de la coopération internationale.

Enfin il ne comprend pas et il n'accepte pas le principe des droits des peuples à disposer d'eux-mêmes, principe dominant à l'assemblée générale de l'ONU, et refuse d'emblée la décolonisation.

Ces positions vont déterminer l'évolution de la politique extérieure future jusqu’à la fin du régime en ce qui concerne les trois questions fondamentales: la sécurité atlantique, la construction européenne et la question coloniale.

En dépit de sa méfiance traditionnelle à l'égard des États-Unis, de la difficulté qu'il a à reconnaître le déclin de la Grande-Bretagne et l'émergence des États-Unis comme grande puissance maritime hégémonique dans l'Atlantique, Salazar va être contraint à en prendre acte sans tarder. Le premier signal sera la signature de l'Accord des Lajes, accord bilatéral de coopération militaire entre le Portugal et les USA en février 1948. La concrétisation, cette fois dans un cadre multilatéral, en sera en dépit de toutes les réticences et résistances de Salazar, l'entrée du Portugal dans l'Alliance Atlantique en avril 1949. L'Accord des Lajes et l'entrée du Portugal à l'OTAN signifient donc la reconnaissance de la nouvelle puissance maritime et la naissance d'une nouvelle alliance qui est, en même temps, la réponse de la politique extérieure portugaise à la nouvelle configuration de la scène internationale de l'aprèsguerre et le retour à la tradition atlantique du Portugal (Teixeira, 1991; Telo, 1996).

Par contre, en ce qui concerne la question européenne, la position portugaise sera assez différente. Elle apparait d'emblée dans les premières hésitations à l'égard du plan Marshall qui conduisent Salazar à refuser le premier exercice en 1947-1948. Il acceptera le deuxième, mais l'évolution de la politique extérieure portugaise face au processus de construction européenne sera marquée par une certaine distanciation et une position double - c'est-à-dire présence dans les organisations économiques et " hostilité " à l'encontre de tout projet d'intégration ou de supranationalité; présence pour des raisons pragmatiques, dans l'Europe économique et rejet, par option stratégique, de toute formule d'Europe politique (Rolo, 1994; Castilho, 2000 ; Pinto, Teixeira, 1998).

L' "option européenne " sera donc la grande nouveauté de la politique extérieure de la démocratie, et la démocratie en était d'ailleurs la condition politique.

$\mathrm{Si}$, à l'intégration dans le système de sécurité atlantique et à l'éloignement à l'égard de la construction européenne, l'on ajoute la défense intransigeante de l'Empire Colonial, allant jusqu'à des conflits de décolonisation qui se poursuivent pendant treize ans dans trois théâtres d'opérations différents, on a la définition des grandes options stratégiques de la politique extérieure portugaise jusqu'à la fin du régime autoritaire (Teixeira, 2004; Schneidman, 2004; Rodrigues, 2002; Martins, 1998; Silva, 1995).

Options qui correspondent avec une clarté absolue au deuxième modèle historique d'insertion internationale du Portugal:

- En premier lieu, la perception d'une antinomie, parfois même d'un dilemme entre l'Europe et l'Atlantique, atteint le paroxysme à la fin de "l'État Nouveau ", précisément dans le débat politique entre les défenseurs des deux options stratégiques pour le pays : les africanistes et les européistes. 
- En deuxième lieu, l'éloignement de l'Europe avec, en corollaire, la prédominance de l'option atlantique et coloniale, prédominance sur le plan politique tout comme dans le domaine économique. Sur le plan politique, on assiste ainsi à un dispositif diplomatico-stratégique qui repose totalement sur le vecteur atlantique, c'est-àdire sur l'intégration à l'OTAN et sur l'alliance privilégiée avec Washington et Londres (en particulier concernant la question Européenne). Dans le domaine économique, s'impose un dispositif géo-économique essentiellement orienté vers l'outremer et les colonies. Même lorsque des considérations pragmatiques obligeaient le pays à se rapprocher des institutions économiques européennes, ce rapprochement continuait à se faire dans le cadre stratégique atlantique et jamais dans le cadre continental. L'AELE et l'entrée du Portugal à l'AELE en sont l'exemple le plus parfait (Griffiths, Lie, 1995; Alípio, 2001; Leitão, 2004).

- En troisième lieu, la diversification constante des alliances extra-péninsulaires; le Portugal sera toujours là où l'Espagne n'est pas. Dans le cadre atlantique le Portugal entre à l'OTAN, l'Espagne demeure en dehors. Dans le cadre européen, le Portugal entre à l'AELE, l'Espagne reste en dehors.

Et enfin, malgré l'interdépendance croissante des relations internationales et de la multilatéralisation progressive du cadre diplomatique, la persistance d'une diplomatie bilatérale, fondée sur le triangle Lisbonne-Madrid-puissance maritime.

Le processus de démocratisation au Portugal a entraîné l'altération de ce cadre d'ensemble de la politique extérieure portugaise. Mais il a entraîné bien plus que cela: le passage à la démocratie et la consolidation démocratique au Portugal et en
Espagne, d'une part, et l'évolution propre de la scène internationale, d'autre part, ont conduit ainsi en douze années à peine (1974-1986) à la disparition de ce modèle historique d'insertion internationale du Portugal, vieux de cinq siècles.

\section{La dimension internationale de la démocratisation}

La fin du régime autoritaire et le processus de transition vers la démocratie, qui a commencé le 25 avril 1974, nécessitent une redéfinition en profondeur de la politique extérieure portugaise, en conformité avec l'esprit du programme du Mouvement des Forces Armées (MFA), redéfinition traduite de façon synthétique par la formule: "démocratisation, décolonisation, développement ". Bien que le programme du MFA ait annoncé qu'il garantissait le respect de tous les engagements internationaux du Portugal, il était clair que ces deux principes simples - démocratisation et décolonisation - impliqueraient nécessairement une réinterprétation de ces mêmes engagements et une modification fondamentale de l'orientation extérieure de l'État portugais.

En 1974 déjà, commencent les négociations en vue de la décolonisation des territoires coloniaux. Et la décolonisation constituera en fait le premier grand défi de politique extérieure pour le nouveau régime (Pinto, 2001 ; Correia, 1999; Cevelló, 1998; Mac Queen, 1997).

Sur cette question, plusieurs conceptions idéologiques s'affrontaient en coulisse: une première tendance suivant les propositions de Spinola dans "Portugal e o Futuro », continuait à insister sur la théorie fédérative; une deuxième tendance, inspirée par Melo Antunes, recherchait la constitution d'un axe 
neutraliste, non-aligné et tiers-mondiste; et enfin Vasco Gonçalves adoptait une tendance pro-soviétique.

Du point de vue politique, ces nuances idéologiques se cristallisaient en deux positions fondamentales : la première soutenait qu'autodétermination ne signifiait pas automatiquement indépendance et insistait, de façon intransigeante, sur le maintien de la souveraineté portugaise jusqu'à un referendum qui devrait décider du destin des territoires coloniaux; la deuxième, au contraire, défendait le principe de l'identité entre autodétermination et indépendance, et réclamait un transfert immédiat du pouvoir aux mouvements de libération, en tant que représentants légitimes des peuples coloniaux.

A la suite d'un processus complexe, qui ne fut pas sans incidences importantes sur la politique intérieure, ce fut la deuxième position qui l'emporta. Au moment où le cessez-le-feu se mettait en place sur le terrain, dans les chancelleries débutaient les premières négociations diplomatiques.

La Guinée Bissau, qui avait déjà déclaré unilatéralement son indépendance en 1973, allait être le premier pays à être reconnu internationalement par l'ancienne puissance coloniale. C'était en août 1974. Et entre août 1974 et janvier 1975 le même processus de transfert du pouvoir aux mouvements de libération allait être suivi avec des variantes propres à chaque cas dans toutes les anciennes colonies portugaises.

En même temps que se déroulait le processus de décolonisation, les relations diplomatiques étaient établies avec l'Union Soviétique, les pays de l'Est et du tiersmonde, à l'exception de l'Albanie et de la Chine, où le processus a rencontré des difficultés importantes qui n'ont été résolues qu'en 1979.
Cependant, la décolonisation, l'ouverture diplomatique et la fin de l'isolement international du pays ne suffisaient pas à elles seules pour définir les nouvelles orientations extérieures de la démocratie portugaise, bien au contraire. En dessous des luttes bruyantes, liées au processus de démocratisation intérieure, se livrait une autre lutte silencieuse concernant les objectifs et les options stratégiques de la politique extérieure portugaise. Entre avril 1974 et janvier 1986, la politique extérieure portugaise oscille entre deux orientations fondamentales qui correspondent à deux phases distinctes : celle de la transition vers la démocratie, coïncidant avec la période pré-constitutionnelle dominée par le processus révolutionnaire; et celle de la consolidation de la démocratie, coöncidant avec la période constitutionnelle marquée par l'institutionnalisation et la stabilisation du régime démocratique (Dulphy, 2003; Telo, 1999a et b).

La période pré-constitutionnelle se caractérise par la lutte autour des options extérieures du pays, par l'exercice des diplomaties parallèles et, de ce fait, par un manque de définition de la politique extérieure. En dépit des luttes, des hésitations et du climat d'incertitude, pendant les gouvernements provisoires et, particulièrement, ceux dominés par les militaires, l'orientation globale de la politique extérieure portugaise tend vers une option tiers-mondiste et vers le développement de relations privilégiées avec les nouveaux pays issus de la décolonisation portugaise. C'est là le dernier avatar, socialisant cette fois-ci, de la thèse de la vocation africaine du Portugal, si chère à Salazar.

La période constitutionnelle, qui débute précisément avec le premier gouvernement constitutionnel, se caractérise par une clarification de la politique extérieure portugaise et par une définition unique et rigoureuse du 
positionnement extérieur du Portugal - qui assume pleinement sa condition de pays occidental à la fois européen et atlantique. Ce seront là les vecteurs essentiels et les véritables options stratégiques du Portugal démocratique.

Le vecteur atlantique a signifié pour le Portugal la permanence des caractéristiques historiques de sa politique extérieure et a joué un rôle important, non seulement au niveau de l'orientation extérieure, mais aussi dans la stabilisation intérieure du pais.

Sur le plan bilatéral, cet atlantisme s'est matérialisé par le resserrement des relations diplomatiques avec les États-Unis et par le renouvellement de l'Accord des Lajes en 1979 et 1983. Par ces accords, le Portugal octroie aux États-Unis les « facilités " des bases des Açores jusqu'en 1991, et reçoit en contrepartie des " aides " dans le domaine économique et militaire (Sá, 2004; Pereira, 1990 ; Magalhães, 1998).

Sur le plan multilatéral, le vecteur atlantique s'est traduit par le maintien et le renforcement de la position du Portugal dans l'Alliance Atlantique et par la redéfinition et le renouvellement de la participation portugaise aux engagements militaires de l'OTAN, que l'effort de guerre en Afrique avait contraint à abandonner depuis les années 60 . En ce qui concerne l'armée, ce renforcement des engagements s'est concrétisé par l'organisation de la Brigade Mixte Indépendante, devenue ensuite la Brigade Aéroportée, qui remplace et réactive l'ancienne Division Indépendante de l'Armée et qui conserve, pour l'essentiel, ses anciens objectifs dans les missions de l'OTAN sur le flanc sud de l'Alliance. Au niveau de la marine et de l'aviation, les missions de patrouille sont renforcées dans le cadre d'IBERLAND, dont le commandement est élevé à la catégorie de Commandement en Chef - CINCIBERLAND - poste qui peut désormais être exercé par un officier portugais (Teixeira, 1999; Telo, 1999c; Rato, 2001).

C'est cependant l'" option européenne " qui constitue la grande nouveauté de la politique extérieure d'après le 25 avril et le grand défi du Portugal démocratique. Une fois dépassées les résistances anti-européennes, d'abord sous la forme de l'option africaine du régime autoritaire, puis de la tentation tiers-mondiste de la période révolutionnaire: le Portugal assume clairement, à partir de 1976, l'" option européenne " - et ceci, désormais, en tant que projet politique et non plus seulement dans une perspective purement économique, comme lors des accords d'association de 1972 (Pinto, Teixeira, 2002, 2004 ; Ferreira, 2003 ; Ribeiro, 1999).

C'est précisément en cette année-là, 1976, que le Portugal commence à se rapprocher du processus de construction européenne avec l'adhésion au Conseil de l'Europe et la signature des Protocoles Additionnels à l'accord de 1972, ce qui constitue, dans une certaine mesure, la phase préliminaire du processus d'adhésion.

Après une série de négociations couronnées de succès qui se sont déroulées dans les capitales européennes de septembre 1976 à février 1977, le premier gouvernement constitutionnel sollicite officiellement l'adhésion du Portugal à la Communauté Économique Européenne. Avec cette demande officielle d'adhésion, les hésitations concernant la formule d'intégration portugaise - statut de pré-adhésion ou formule dite d' " association privilégiée " - sont surmontées et l'" option européenne " se concrétise clairement. C'est une option stratégique qui marquera de façon décisive 
l'avenir du pays et qui vient compléter la dimension internationale de la consolidation démocratique portugaise.

La décision du gouvernement et le choix de cette option stratégique répondaient à deux objectifs: en premier lieu, la consolidation de la démocratie que garantissait l'entrée du Portugal dans la Communauté; et, en deuxième lieu, la modernisation et le développement économique que la communauté allait favoriser (Magone, 2004; Lopes E. R., 2003; Barreto, 1999; Lopes J. S. 1993).

La demande d'adhésion allait être suivie d'un long et complexe processus de négociation, qui allait se prolonger pendant plus d'une décennie et culminer en juin 1985 avec la signature du Traité d'adhésion du Portugal à la CEE. À partir du $1^{\text {er }}$ janvier 1986, le Portugal devient membre de plein droit de la Communauté Européenne et, au cours de cette même année, il signe l'Acte Unique Européen.

Bien que le développement de relations et de liens d'amitié et de coopération avec les nouveaux pays d'expression officielle portugaise continue de constituer une préoccupation pour le Portugal et que, depuis 1976, non seulement les gouvernements, mais également les présidents de la République n'aient épargné aucun effort diplomatique pour améliorer les relations avec les Pays d'Expression Officielle Portugaise (PALOPS) (Marchueto, 2003 ; Lamego, 2001 ; Léonard, 1988; Enders, 1994a et b), la vérité est que l'option stratégique centrale de la démocratie portugaise passe par l'européisation de sa politique extérieure.

En fait, l'Union européenne, l'OTAN et les relations post-coloniales auxquelles la Communauté des Pays de Langue Portugaise (CPLP) donne un cadre multilatéral, constituent aujourd'hui, les axes fonda- mentaux et les priorités stratégiques de la politique extérieure et de la présence internationale du Portugal.

\section{Continuité et changement dans la politique extérieure portugaise}

Dans une perspective de longue durée historique, il faut prendre en considération, finalement, deux considérations finales sur les deux questions posées à l'origine.

En premier lieu, concernant la dimension temporelle du processus de démocratisation: existe-t-il ou non une coïncidence chronologique entre les processus de transition et de consolidation au niveau intérieur et au niveau extérieur? S'il est vrai que cette coïncidence se vérifie pour le processus de transition, on ne peut en dire autant pour ce qui est de la consolidation démocratique. Dans le premier cas, la fin de la transition et le début de la période constitutionnelle, au niveau interne, vont de pair avec la clarification de la position du Portugal en tant que pays occidental à la fois atlantique et européen. Dans le deuxième cas, la fin de la consolidation intérieure ne coïncide pas avec la consolidation sur le plan international. Si on peut considérer la première comme achevée en 1982, avec la révision constitutionnelle et la loi de la défense nationale et des forces armées, pour la deuxième il a fallu attendre 1986 avec l'adhésion du Portugal à la Communauté Européenne.

En deuxième lieu la question de la continuité ou de la rupture dans la politique extérieure portugaise avant et après la démocratie. Quels sont les éléments permanents et quels sont les changements?

Les lignes permanentes, qui se sont maintenues, découlent des éléments structurels et géopolitiques et sont essentiellement les 
domaines d'intérêt stratégique du Portugal: l'Atlantique, l'Europe et les relations postcoloniales.

Les changements sont, au moins, au nombre de quatre.

- Premièrement, la logique d'antinomie entre l'Europe et l'Atlantique a changé. Cette logique n'a plus de sens aujourd'hui, et ses termes, non seulement, ne sont plus contradictoires mais sont même complémentaires. Pour la politique extérieure portugaise, le fait d'être un pays atlantique peut représenter une valeur ajoutée en Europe, tout comme le fait d'être européen peut apporter une valeur ajoutée dans l'Atlantique et, en particulier, dans l'Atlantique sud où se situent les relations post-coloniales.

- Deuxièmement, dans le binôme Europe-Atlantique, l'équation géopolitique se maintient, mais les priorités stratégiques sont inversées; traditionnellement, le Portugal avait une priorité atlantique et coloniale, et lorsque le poids du vecteur maritime était excessif, il recherchait des compensations continentales. Aujourd'hui, au contraire, la priorité c'est l'Europe et l'Union Européenne et, pour accroître son pouvoir, le Portugal s'efforce de revaloriser et de renforcer sa position atlantique et ses relations post-coloniales.

- Troisièmement, l'instauration de la démocratie au Portugal et en Espagne a entraîné un rapprochement des positions internationales des deux états de la péninsule. En 1974-1975 le Portugal décolonise. En 1979 l'Espagne se rapproche de l'AELE. En 1982, elle intègre la structure politique de l'OTAN. En 1986, le Portugal et l'Espagne entrent conjointement dans la Communauté Économique Européenne. Quatre ans plus tard, toujours conjointement, ils entrent aussi dans l'UEO. En 1997 l'Espagne entre dans la structure militaire de l'OTAN. Cela signifie que, non seulement la position géo-économique portugaise s'est continentalisée avec l'entrée dans la communauté européenne, mais que les positions stratégiques du Portugal et de l'Espagne se sont rapprochées progressivement jusqu'à coïncider. En d'autres termes, le Portugal et l'Espagne partagent aujourd'hui, pour la première fois, les mêmes alliances extra-péninsulaires : UE/OTAN/UEO.

- Quatrième et dernier point, à la suite de l'interdépendance croissante des relations internationales et l'importance accrue des structures diplomatiques multilatérales, la diplomatie portugaise enregistre un déclin progressif du bilatéralisme au profit du multilatéralisme, ce qui amène le Portugal à être présent dans les organisations multilatérales correspondant à ses zones d'intérêt stratégique: l'Union européenne pour l'Europe, I'OTAN pour l'Atlantique et la CPLP pour les relations post-coloniales.

Dans une perspective à long terme ces changements, qu'il n'est pas trop audacieux de qualifier de radicaux, signifient la fin du modèle historique d'insertion internationale du pays et représentent les premières tentatives de la politique extérieure portugaise pour se construire un nouveau modèle fondé sur l'européisation. 


\section{Références}

Alípio E.S., O Processo Negocial de Adesão de Portugal à EFTA 1956-1960, thèse non publiée, presentée à la Faculté de Sciences Sociales et Humaines, Universidade Nova Lisboa, 2001.

Barreto A., "Portugal: Democracy trough Europe ", in Anderson J. J. (edited by), Regional Integration and Democracy, New York Oxford, Rowman \& Littlefeld, 1999.

Carrilho, M. et al., Portugal na Segunda Guerra Mundial, Lisbonne, D. Quixote, 1989.

Castilho, J.M.T., A Ideia de Europa no Marcelismo 1968-1974, Lisbonne, Ediçōes Afrontamento, 2000.

Cevelló J. S., El Último Império Occidental: La Descolonización Portuguesa, Mérida, UNED, 1998.

Correia P. P., " Descolonização ", in Brito J.M.B., Do Marcelismo ao fim do Império, Lisbonne, Círculo dos Leitores, 1999.

Delgado I. Portugal e a Guerra Civil de Espanha, Lisbonne, Publicaçōes Europa-América, 1981.

Dulphy A., Léonard Y (dir.), De la dictature à la démocratie: voies ibériques, Bruxelles, Peter-Lang, 2003.

Enders A., Histoire de l'Afrique Lusophone, Éditions Chandeigne, Paris, 1994a.

Enders A., "Géopolitques des Mondes Lusophones ", Lusotopiei, no 1-2, Paris, $1994 \mathrm{~b}$.

Ferreira, J. M., "A estratégia para a adesão de Portugal às Instituiçōes Europeias ", in Ribeiro M. M. T., Melo A. M. B. de, Porto M.C.L. (dir.), Portugal e a Construçāo Europeia, Coimbra, Almedina, 2003.

Griffiths R., Lie B., "Portugal e a EFTA 1969-1973 ", in Portugal e a Europa 50 anos de integração, Lisbonne, Centro de Informaçáo Jacques Delors, 1995.

Huntington S. P., The Third Wave, Oklahoma, University of Oklahoma Press, 1991.

Lamego J., "A emergência da CPLP e as suas consequências ", in Ferreira J. M., Política Externa e Política de Defesa do Portugal Democrático, Lisbonne, Ediçōes Colibri, 2001.

Leitão N. A., " O convidado inesperado: Portugal e a fundação da EFTA 1956-1960 ", Análise Social, no 171, Lisbonne, 2004.

Léonard Y., La Lusophonie dans le Monde, Paris, La Documentation Française, 1988.

Linz J. J., Stepan A. The Breakdown of Democratic Regimes, Baltimore, Johns Hopkins Press, 1978.

Lopes E. R., "O processo de integração de Portugal nas Comunidades Europeias-uma avaliação geral década e meia depois ", in Ribeiro, M.M.T., Melo A.M.B de, Porto M.C.L. (dir.), Portugal e a Construção Europeia, Coimbra, Almedina, 2003.

Lopes J. S. Portugal and EC Membership Evaluated, Londres, New York, Pinter Publishers, 1993.

Macedo J. B. de, História Diplomática de Portugal. Constantes e Linhas de Força, Lisbonne, IDN, 1987.

MacQueen N., The Portuguese Decolonization. Metropolitan Revolution and the Dissolution of Empire, Londres, New York, Longman, 1997.

Magalhães J. C., "Portugal e os Estados Unidos-relaçōes no domínio da defesa ", Estrateggia, n 4, Lisbonne, 1988.

Magone J. M., The Developing Place of Portugal in the European Union, Brunshwick, Londres, Tranaction Publishers, 2004.

Marchueta M. R., A CPLP e o seu enquadramento, Lisboa, Ministério dos Negócios Estrangeiros, 2003.

Martins F., "A Política Externa do Estado Novo, o Ultramar e a ONU ", Penélope, n 18, Lisbonne, 1998.

Melo A. M. B. de, Porto M. C. L. (dir.), Portugal e a Construção Europeia, Coimbra, Almedina, 2003.

Oliveira C., Salazar e a Guerra Civil de Espanha, Lisbonne, O Jornal, 1987.

Pereira B. F., «Continuidade na Mudança : as relaçōes entre Portugal e os Estados Unidos », Politica Internacional, $n^{\circ} 2$, Lisbonne, 1990.

Pinto A. C., O Fim do Império Português, Lisbonne, Livros Horizonte, 2001.

Pinto A. C., Teixeira N. S., " Portugal e a Unificação Europeia ", Penélope, no 18, Lisbonne, 1998.

Pinto A. C., Teixeira N. S., "From Africa to Europe: Portugal and European integration ", in Pinto A.C., Teixeira N. S. (eds.), Southern Europe and the Making of the European Union, New York, SSM, Columbia University Press, 2002.

Pinto A. C., Teixeira, N. S., « From Atlantic Past to European destiny ", in Kaiser W, Elvert J. (eds.), European Union Enlargement. A comparative History, Londres, New York, Routledge, 2004.

Pridham G., "The international dimensions of democratisation ", chapitre neuf de The Dynamics of Democratization. A Comparative Approach. Londres, Continuum, 2000.

Pridham G., Encouraging Democracy. The International Context of Regimen Transition in Southern Europe, Leicester, Leicester University Press, 1991. 
Pridham G., Herring E., Building Democracy. The International Dimension of Democratization in Eastrn Europe, Leicester, Leicester University Press, 1997.

Rato V., "A Aliança Atlântica e a Consolidação da Democracia ", in Ferreira J. M.(dir.), Política Externa e Polttica de Defesa do Portugal Democrático, Lisbonne, Ediçōes Colibri, 2001.

Ribeiro M. M. T., "Le Portugal et le nouveau défi européen ", in Fédérations ou Nations, a cura de Ariane Landuyt, Paris, Éditions SEDES, 1999.

Rodrigues L. N., Salazar e Kennedy: a crise e uma aliança, Lisbonne, Editorial Notícias, 2002.

Rolo F., Portugal e o Plano Marshall, Lisbonne, Editorial Estampa, 1994.

Rosas F. (dir.), Portugal e a Guerra Civil de Espanha, Lisbonne, Ediçōes Colibri, 1998.

Rosas F., Portugal entre a Paz e a Guerra, Lisbonne, Editoral Estampa, 1990.

Sá T. M., Os Americanos na Revolufão Portuguesa (1974-1976), Lisbonne, Notícias Editorial, 2004.

Schmitter P., "O contexto internacional da democratização contemporânea ", chapitre VIII de Portugal: do Autoritarismo à Democracia, Lisbonne, Imprensa de Ciências Sociais, 1999.

Schmitter P., O’Donnell G., Whitehead L., Transitions from Autoritarian Rule: Prospects to Democracy, Baltimore, Johns Hopkins Press, 1986.

Schneidman W.W., Engaging Africa: Washington and the Fall of Portugal's Empire, New York, Oxford, University Press of América, 2004.

Silva A. E. D., "O litígio entre Portugal e a ONU ", Análise Social, n 30, Lisbonne, 1995.

Stone G., The Oldest Ally: Britain and Portuguese Connection, Londres, Boydell, 1994.

Teixeira N. S., From Neutrality to Allignment: Portugal and the Fondation of the Atlantic Pact, Florence, Institut Universitaire Européen, 1991.

Teixeira N. S., "Portugal na NATO 1949-1999 ", Nação e Defesa, no 89, 1999.

Teixeira N. S., "Between Africa and Europe: Portuguese Foreign Policy 1890-1986 ", in Pinto A.C. (ed.) Contemporary Portugal, New York, SSM, Columbia University Press, 2003.

Teixeira N.S., "Portugal e as Guerras da Descolonizaçáo " in Barata M.T., Teixeira N.S., Nova História Militar, volume 4, Lisbonne, Círculo de Leitores, 2004.

Telo A. J, Portugal na Segunda Guerra, Lisbonne, Perspectivas e Realidades, 1987.

Telo A. J, Portugal na Segunda Guerra, 2 volumes, Lisbonne, Veja, 1991.

Telo A. J., Portugal e a NATO. O reencontro da tradiçāo atlantica, Lisbonne, Cosmos, 1996.

Telo A. J., " As Relaçóes Internacionais da Transição ", in Brito J. M. B., Do Marcelismo ao fim do Império, Lisbonne, Círculo dos Leitores, 1999a.

Telo A. J., "A Revoluçāo e a posição de Portugal no Mundo ", in Rosas F. (dir.), Portugal e a Transição para a Democracia, Lisbonne, Ediçōes Colibri, 1999b.

Telo A. J., " Portugal e a Nato (1949-1976) ", Nação e Defesa, no 89, Lisbonne, 1999c.

Telo A. J., A Neutralidade Portuguesa e o Ouro Nazi, Lisbonne, Quetzal, 2000

Whitehead L. The International Dimension of Democratization: Europe and Americas, Oxford, Oxford University Press, 1996. 